\title{
The Expression and Significance of CyclinD2 and Bcl-2 in Diffuse Large B-cell Lymphoma
}

Qiushuang $\mathrm{Ma}^{1 \#}$, Huijuan Geng ${ }^{2 \#}$, Yafei Zhao ${ }^{3}$, Jirui Sun ${ }^{1}$, Xing Zhou ${ }^{1}$, Haiying Yao ${ }^{4 *}$, Jinku Zhang ${ }^{1 *}$

${ }^{1}$ Department of Pathology, Baoding First Central Hospital, Baoding 071000, Hebei province, China;

${ }^{2}$ Department of Laboratory Medicine, Baoding People's Hospital, Baoding 071000, Hebei province, China;

${ }^{3}$ Department of Orthopedics, Baoding First Hospital of Traditional Chinese Medicine, Baoding 071000, Hebei province, China

${ }^{4}$ Department of Hematology, Baoding First Central Hospital, Baoding 071000, Hebei province, China

*Jinku Zhang and Haiying Yao both are the Corresponding authors and contributed equally to this work.

"Qiushuang Ma and Huijuan Geng both are the first authors and contributed equally to this work.

Abstract: Objective: To study the expression and significance of cyclinD2 and Bcl-2 in diffuse large B-cell lymphoma. Methods: This project used immunohistochemical methods to detect the expression of cyclinD2 and Bcl-2 in 120 cases of diffuse large B-cell lymphoma and 80 cases of reactive hyperplasia of lymphoid tissue. The materials were collected from the hospital from January 2018 to 2020. In March 2015, 120 patients had lymphoma tissue removed during the month of surgery. The postoperative pathological diagnosis was DLBCL. Another 80 cases of lymphoid hyperplasia (RLH) tissues were selected as controls. Results: CyclinD2 and BCL-2 expression were not statistically different in patients with diffuse large B-cell lymphoma in different ages, genders, locations, tissue types, and degree of differentiation; but statistically significant in different Ann Arbor stages, immunotypes, IPI index and first treatment efficacy. Conclusion: This research not only has important theoretical value, but also important economic value and social significance.

Keywords: CyclinD2; Bcl-2; Lymphoma

Publication date: November, 2020

Publication online: 30 November, 2020

"Corresponding author: Haiying Yao, haiyingyaobd @163.com; Jinku Zhang, 843561234@qq.com

Diffuse large B-cell lymphoma is the most common type of non-Hodgkin's lymphoma (NHL), accounting for almost one-third of all cases. The cause is unknown, and it is related to immunodeficiency and environmental factors. The most typical manifestation of clinical symptoms is painless, progressive swelling of the superficial lymph nodes. The swelling has smooth surface, hard texture, and feels like a ping pong ball or the hardness of the tip of the nose. It may cause damage to the digestive, urinary, nervous and other systems, and threaten life ${ }^{[1]}$. China is a country with a large population. Diffuse large B-cell lymphoma in China accounts for about $50 \%$ of Hodgkin's lymphoma. Rituximab combined with cyclophosphamide, doxorubicin, vincristine and prednisone (CHOP) can cure $76 \%$ of CD20positive patients, and about $30 \%$ of diffuse large B-cell lymphomas are refractory, relapsing after sexual intercourse or chemotherapy. $1 \%$ to $2 \%$ of diffuse large B-cell lymphomas is negative for CD20 and is ineffective for rituximab treatment. These patients are often accompanied by involvement of extranodal organs, and the prognosis is poor due to chemotherapy resistance. The median survival period ranges from several months to several years, and patients are prone to early relapse. Therefore, this paper will analyze the expression and significance of cyclinD2 and Bcl-2 in diffuse large B-cell lymphoma. Now the report is as follow ${ }^{[2]}$. 


\section{Materials and Methods}

\subsection{General Materials}

From January 2018 to March 2020, 120 cases of lymphoma tissue were collected in the hospital. The postoperative pathological diagnosis was DLBCL, and 80 cases of reactive lymphoid hyperplasia (RLH) tissue were selected as controls. All patients with diffuse large B-cell lymphoma received no treatment before surgery and had complete follow-up data.

\subsection{Methods}

The differences in expression of cyclinD2 and Bcl-2 in diffuse large B-cell lymphoma tissue and lymphoid tissue reactive hyperplasia were analyzed by immunohistochemistry. The relationship between cyclinD2 and Bcl-2 and clinicopathological characteristics was analyzed, and the relationship between cyclinD2 and Bcl-2 was explored. Bcl-2 is related to the occurrence and development of diffuse large B-cell lymphoma.

\subsection{Observation Indicators}

To detect the relationship between cyclinD2 and Bcl-

2 , and to explore the relationship between cyclinD2 and $\mathrm{Bcl}-2$ in the occurrence and development of diffuse large B-cell lymphoma. The therapeutic effect of cyclinD2 and Bcl-2 on diffuse large B-cell lymphoma was observed.

\subsection{Statistical Methods}

The data of this study was statistically processed by spss. 20.0 software, and the measurement data is expressed in \%.

\section{Results}

\subsection{CyclinD2, Bcl-2 Expressions}

CyclinD2 and Bcl-2 have differential expression of lymphoid tissue reactive hyperplasia in diffuse large B-cell lymphoma tissue, and the difference is statistically significant, see Table 1 .

\subsection{Clinicopathological Characteristics}

The expression of cyclinD2 and Bcl-2 has nothing to do with the age, sex, location, tissue type and degree of differentiation of diffuse large B-cell lymphoma; it is related to the efficacy obtained through the first treatment, IPI index and immune type. For details, see Table 2.

Table 1. Comparison of cyclinD2 and Bcl-2 expression between the two groups $[n(\%)]$

\begin{tabular}{ccc}
\hline Group & $\boldsymbol{n}$ & cyclinD2 \\
\hline Diffuse large B-cell lymphoma group & 87 & $29(32.83)$ \\
Lymphoid tissue reactive hyperplasia group & 23 & $54(60.92)$ \\
\hline
\end{tabular}

Table 2. The expression and clinicopathological characteristics of cyclinD2 and Bcl-2 in diffuse large B-cell lymphoma

\begin{tabular}{|c|c|c|c|c|c|c|c|c|c|c|}
\hline \multirow{2}{*}{ Indicator } & & \multirow{2}{*}{$\mathbf{n}$} & \multicolumn{2}{|c|}{ cyclinD2 } & \multirow{2}{*}{$\chi^{2}$} & \multirow{2}{*}{$P$} & \multicolumn{2}{|c|}{ Bcl-2 } & \multirow{2}{*}{$\chi^{2}$} & \multirow{2}{*}{$P$} \\
\hline & & & Positive & Negative & & & Positive & Negative & & \\
\hline \multirow[t]{2}{*}{ Age (y.o.) } & $\geq 40$ & 55 & 18 & 37 & \multirow{2}{*}{0.025} & \multirow{2}{*}{0.874} & 36 & 19 & \multirow{2}{*}{0.396} & \multirow{2}{*}{0.254} \\
\hline & $<40$ & 34 & 13 & 21 & & & 20 & 14 & & \\
\hline \multirow[t]{2}{*}{ Gender } & Male & 41 & 15 & 36 & \multirow{2}{*}{0.026} & \multirow{2}{*}{0.165} & 36 & 5 & \multirow{2}{*}{0.258} & \multirow{2}{*}{0.336} \\
\hline & Female & 47 & 22 & 25 & & & 25 & 14 & & \\
\hline \multirow[t]{2}{*}{ Site } & Extranodal & 52 & 13 & 16 & \multirow{2}{*}{1.078} & \multirow{2}{*}{0.369} & 58 & 9 & \multirow{2}{*}{0.246} & \multirow{2}{*}{0.259} \\
\hline & Intranodal & 36 & 15 & 19 & & & 45 & 16 & & \\
\hline \multirow[t]{2}{*}{ Tissue Type } & ImmunoblastCentral blast & 12 & 4 & 25 & \multirow{2}{*}{0.065} & \multirow{2}{*}{0.897} & 25 & 10 & \multirow{2}{*}{0.058} & \multirow{2}{*}{0.587} \\
\hline & & 78 & 25 & 26 & & & 26 & 25 & & \\
\hline \multirow[t]{2}{*}{ Degree of Differentiation } & High & 50 & 15 & 36 & \multirow{2}{*}{0.016} & \multirow{2}{*}{0.842} & 14 & 18 & \multirow{2}{*}{0.415} & \multirow{2}{*}{0.367} \\
\hline & Low & 39 & 14 & 25 & & & 36 & 26 & & \\
\hline \multirow[t]{2}{*}{ Ann Arbor Stage } & I-I & 54 & 16 & 45 & & & 24 & 19 & & \\
\hline & I-IV & 36 & 15 & 16 & 0.036 & 0.000 & 34 & 24 & 16.254 & 0.000 \\
\hline Immunophenotyping & non-GCB Type & 45 & 6 & 45 & 16321 & 0000 & 28 & 26 & 25236 & 0003 \\
\hline & GCB Type & 42 & 24 & 17 & 10.321 & 0.000 & 45 & 13 & 25.250 & 0.003 \\
\hline IPI Index & Low & 41 & 7 & 36 & & & 46 & 25 & & \\
\hline & High & 48 & 23 & 32 & 10.252 & 0.009 & 48 & 11 & 10.008 & 0.000 \\
\hline First Treatment Efficacy & Complete Remission & 52 & 16 & 47 & & & 53 & 28 & & \\
\hline & Incomplete Remission & 36 & 18 & 38 & 0.232 & 0.035 & 41 & 6 & 15.258 & 0.012 \\
\hline
\end{tabular}




\section{Discussion}

Diffuse large B-cell lymphoma is a malignant tumor of the circulatory system and belongs to the category of lymphoma. Lymphoma is divided into Hodgkin's lymphoma and non-Hodgkin's lymphoma, and diffuse large B-cell lymphoma is the most common lymphatic system tumor in adults, that is, it belongs to the most common pathological type of nonHodgkin's lymphoma. It accounts for $30 \%-40 \%$ of all non-Hodgkin's lymphomas. The typical clinical manifestations of diffuse large B-cell lymphoma are painless, progressive lymphadenopathy, often accompanied with fever, fatigue, and night sweats. Among them, lymphoma in $40 \%$ of patients can also originate in lymph nodes. The most common sites involved are the gastrointestinal tract, stomach or ileocecal area. It often appears in other parts too, but they are unlikely to originate in the bone marrow or directly involve the blood ${ }^{[3]}$. Diffuse large B-cell lymphoma is more common in elderly people over 60. Clinically, "rapid increase in painless mass" is a typical symptom. It can also cause varying degrees of nosebleeds and difficulty swallowing. The prognosis is usually unsatisfactory ${ }^{[4]}$ It can also be seen in some children. The patient responded better to chemotherapy. Co-administration with cyclinD2 and $\mathrm{Bcl}-2$ can greatly improve the survival rate of patients.

Bcl-2 can inhibit cell death caused by various cytotoxic factors. Overexpression of Bcl-2 can enhance the observed resistance of cells to most cytotoxins $^{[5]}$. Since Kerr put forward the concept of apoptosis in 1972, people have conducted extensive and in-depth research on the phenomenon of apoptosis. However, so far, the molecular and biochemical mechanisms of apoptosis have not been fully understood, and the initial understanding mainly comes from the study of the Bcl-2 gene family ${ }^{[6]}$. It is known that the process of apoptosis can be divided into three phases: induction phase, action phase and degradation phase. In the induction phase, cells receive various signals to trigger various effects. After entering the action phase, the cells enter irreversible programmed death after certain regulatory molecules decided the fate (survival/death) of cells. These regulatory molecules include a series of proto-oncogenes and their inhibitory effects on oncogenes, among which the Bcl-2 family plays a decisive role. During the degradation phase, visible apoptosis occurs.

CyclinD2 can activate corresponding cell cycle protease and form a complex, guide RB phosphorylation inactivation, promote up-regulation of gene expression, and participate in DNA synthesis. Bcl2 gene (ie, B-cell lymphoma/leukemia 2 gene) is an oncogene that has an inhibitory effect on cell apoptosis. In recent years, some studies have begun to reveal the mechanism of this effect. The Bcl-2 protein family discovered so far can be divided into two categories according to their functions. The first type is Bcl-2, which can inhibit cell apoptosis, such as mammalian Bcl-XL, Bcl-W, Mcl-1, A1, nematode Ced-9, and vaccinia virus E1B119kD, etc., while the other type has the ability to promote cell apoptosis, such as Bax, Bcl-Xs, Bad, Bak, Bik/Nbk, Bid and Harakiri. Initially, Bcl-2 was found to inhibit cell death in blood lymphocytes, and this effect was also found in other cells. However, recent studies have found that in addition to these, there are Bcl-2 insensitive apoptosis pathways. The combination of the two categories can effectively alleviate and treat diffuse large B-cell lymphoma.

Through the research above, the theoretical basis and technical support are provided for the discovery of new treatment targets for diffuse large B-cell lymphoma. The research not only has important theoretical value, but also important economic value and social significance.

\section{Acknowledgement}

Key Laboratory of Molecular Pathology and Early Diagnosis of Tumor in Hebei Province

\section{References}

[1] Wei LK, Jun W, Fei Z. Expression and significance of CyclinD2, BCL-2, Survivin in diffuse large B-cell lymphoma[J]. Zhejiang Practical Medicine, 2019, 24(1): 6-8.

[2] Ya HM, Li HS. The expression and clinical significance of Bc1-6, Bc1-2 and NFkB in diffuse large B-cell lymphoma. Chinese Journal of Laboratory Diagnosis, 2017, 21(3): 510.

[3] Wen TH, Hui ZZ, Xiao YZ, et al. The expression of CyclinD2 in diffuse large B-cell lymphoma and its clinical significance[J]. Chinese Journal of Clinical and Experimental Pathology, 2018, 34(1): 27

[4] Xiao YT, Hui P. Research progress on the incidence and survival of extranodal diffuse large B-cell lymphoma[J]. Medical Recapitulate, 2016, 22(15): 2972 
[5] Ming JH, Guo WL, Xiao MO. Clinicopathological significance of the co-expression of MYC and BCL-2 protein in diffuse large B-cell lymphoma[J]. Medical Science Journal of Central South China, 2017, 45(3): 283
[6] Wen YW, Xiao FH, Ying MW, et al. Discussion on the standardization of immunohistochemistry technology $[\mathrm{J}]$. Chinese Journal of Cellular and. Molecular Immunology, 2011, 27(8): 927 